\title{
A Rare Case of Tubal Malignancy with Atypical Features
}

\author{
Dr. Kakarla Jaya Prada Devi ${ }^{1}$, Associate Professor \\ Dr. V. Sandeep ${ }^{2}$, MD Assistant Professor, \\ Dr. Prashanthi Vemulapalli ${ }^{3}$, Assistant. Professor, \\ Dr. Adusumilli Padmaja ${ }^{4}$, Assistant Professor, \\ Dr. Kalyan Chakravarthy ${ }^{5}$, Professor \\ ${ }^{1,3,4}$ (Dept. of OBG, Dr. Pinnamaneni Siddhartha Institute of Medical Sciences \& Research Foundation, \\ Chinoutpalli, AP, India) \\ ${ }^{2}$ (Dept. of Radiology, Dr. Pinnamaneni Siddhartha Institute of Medical Sciences \& Research Foundation, \\ Chinoutpalli, AP, India) \\ ${ }^{5}$ (Dept. of Pathology, Dr. Pinnamaneni Siddhartha Institute of Medical Sciences \& Research Foundation, \\ Chinoutpalli, AP, India)
}

\begin{abstract}
Fallopian tube malignancy is the rarest among genital malignancies ranging to $0.3 \%{ }^{* 1 .}$ There are no specific symptoms related to fallopian tube malignancy, therefore the diagnosis mainly depends on imaging \& histopathology. We are reporting a case of fallopian tube cancer in a 40 year multiparous lady who presented with vague lower abdominal pain and no other symptoms suggestive of genital malignancies. Therefore women presenting with symptoms like abdominal pain, white discharge per vaginum should be subjected to thorough clinical examination \& relevant investigations to arrive at a proper diagnosis at the earliest time possible, to give better prognosis to the patient.
\end{abstract}

Keywords: Fallopian tube malignancy, atypical, reproductive age.

\section{Introduction}

Fallopian tube malignancy is the rarest among genital tract malignancies, incidence ranging to $0.3 \%$. The peak incidence is in the ages of 60 and 64 years, with the mean age of incidence being 55 years (age range: 17 to 88 years) $)^{2}$.Most of the cases are postmenopausal. Common risk factors are nulliparity, genetic $\left(\mathrm{BRCA}_{1} \& \mathrm{BRCA}_{2} \text { mutation }\right)^{4}{ }^{5}{ }^{6}$ \&environmental factors. Common symptoms range from abnormal vaginal bleeding, excessive watery discharge $\mathrm{P} / \mathrm{V}$, vague lower abdominal pain or discomfort. Latzko's triad of symptoms, consisting of intermittent profuse serosanguinous vaginal discharge, colicky pain relieved by discharge and abdominal or pelvic mass has been reported in $15 \%$ of cases ${ }^{7}$. Hydrops tubae profluens, a pathognomonic feature, implies intermittent discharge of clear or blood-tinged fluid spontaneously or on pressure followed by shrinkage of an adnexal mass, occurs in 5\% of patients. The preoperative diagnosis is extremely rare, Most of the cases are diagnosed only during laparotomy ${ }^{8,9}$ due to their nonspecific presentation. We are reporting a rare case of tubal malignancy in a 40 years multiparous lady with no identified risk factors who presented with vague lower abdominal pain on left side.

\section{Case Report}

Mrs X aged 40 years reported to Gynaec OPD at Dr. PSIMS \& RF with complaints of vague lower abdominal pain on left side of 2 months duration. Pain was dull aching in nature, intermittent \&non radiating. There was no h/o abnormal white discharge per vaginum, bowel \& bladder complaints. No H/O dyspareunia, no $\mathrm{h} / \mathrm{o}$ weight loss or loss of appetite. She is $\mathrm{P}_{2} \mathrm{~L}_{2}$, tubectomised. Her menstrual cycle were regular, LMP was 4 days back. NoH/O Dysmenorrhoea.

O/E: GC was good, vitals stable, CVS/RS - NAD, P/A: soft, nontender, central obesity +, VVE normal, P/S - cx vagina healthy, no bleeding or abnormal discharge. Bimanual examination: uterus AV \& normal size, firm, mobile, acystic mass about $6 \times 5 \mathrm{~cm}$ was felt in the left fornix very close to the uterus, nontender. USG: Irregular heterogenous lesion in left adnexa measuring 6.7 x $6.0 \mathrm{~cm}$ in close proximity with uterus (subserous fibroid//ovarian mass). No obvious internal vascularity seen within the lesion. Both the ovaries were not visualized. Minimal free fluid was seen in the POD.CA 125 was 5 IU. Blood investigations WNL .Papsmearwas normal.

MRI was done to confirm the origin and characterisation of tumor which revealed a well defined irregular lesion in the left adnexal region measuring $7.0 \mathrm{~cm}$ X $6.3 \mathrm{~cm}$. Few cystic lesions were seen surrounding the superior portion of the lesion. These could be tumoral cysts or hydrosalpinx. Minimal fluid was seen surrounding the 
lesion. This was not significantly enhancing after the contrast study. Left ovary was not separately seen from the lesion. For this left adnexal lesion, the imaging possibilities were

1. Ovarian tumour (benign/malignant)

2. Subserosal/broad ligament fibroid.

Case was posted for laparotomy.

\section{Intraoperative findings:}

Clear peritoneal fluid of about $100 \mathrm{ml}$ obtained and sent for cytology. Uterus was normal in size. Right side tube \& ovary were normal. Left side ovary was normal. The medial $2-3 \mathrm{~cm}$ of left fallopian tube was normal. The lateral part of the left tube including fimbrial end was replaced by an irregular soft fragile mass of about 7 × $6 \mathrm{cms}$.

Frozen section revealed, papillary Serouscystoadenocarcinoma of fallopian tube. So we proceeded with TAH + BSO. Omental biopsy was taken. Patient recovered well postoperatively. Patient was referred to medical oncologist for further followup and treatment.

HPE: revealed no malignant cells in ascitic fluid.omentum, uterus \& ovaries were normal. Left fallopian tube: Multiple sections showed normal fallopian tube at medial end \& adjacent dysplastic epithelium with left papillary serouscystadenocarcinoma towards fimbrial end.

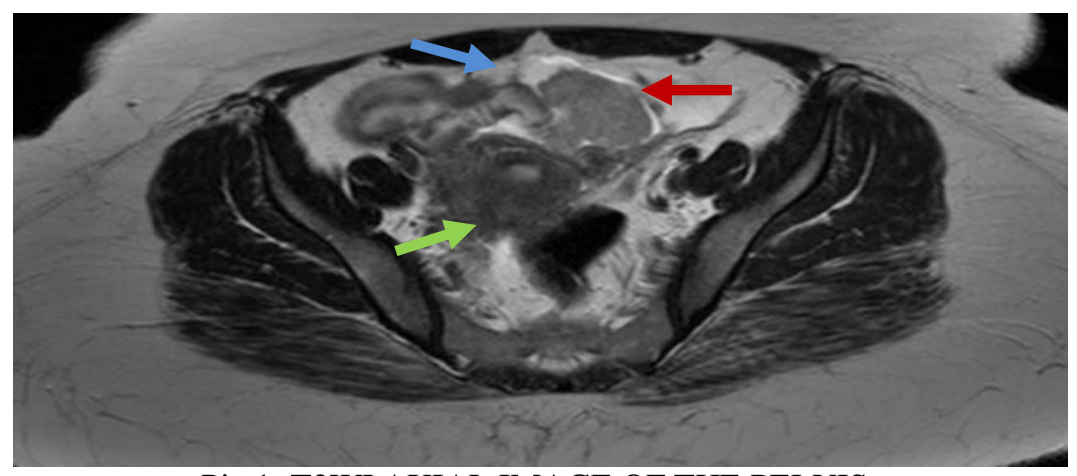

Pic 1: T2WI AXIAL IMAGE OF THE PELVIS.

Red arrow showing tumour separate from the broad ligament $\&$ uterus (green arrow), could be sub serosal location. Contained fluid (blue arrow) surrounding the tumour could be tumoural cyst or hydrosalpinx.

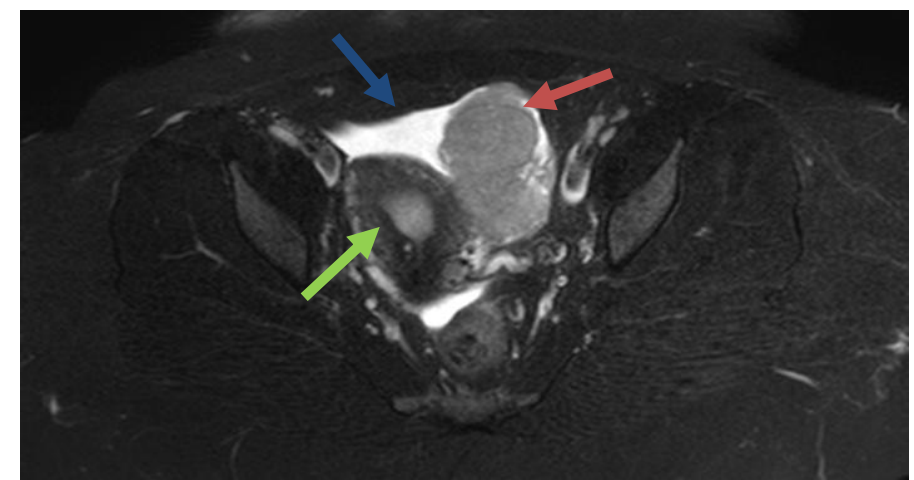

Pic 2: T2WI FATSAT AXIAL IMAGE OF PELVIS:

Pic showing tumour (red arrow), mildly thickened endometrial cavity (green arrow) and ascites (blue arrow)



Pic 3: Green arrow showing uterus, yellow arrow showing mass. 


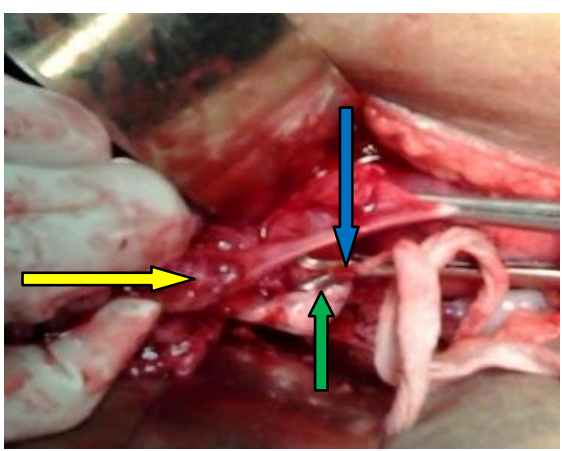

Pic 4: Yellow arrow showing the mass at fimbrial end, blue arrow showing normal medial end of tube, green arrow showing normal ovary.

\section{Discussion}

Fallopian tube malignancy is the rarest among genital malignancies. Risk factors are similar with ovarian malignancy like nulliparity, genetic mutations of BRCA1 \& BRCA2, environmental factors like asbestos exposure \& postmenopausal age. Symptoms range from abnormal vaginal bleeding, excessive watery discharge $(50 \%)$, vague abdominal pain \&distention. Most of the patients present with nonspecific symptoms like dull abdominal pain leading to delay in presentation \& diagnosis. In the above case there were no identifiable risk factors \& she presented with vague pain in the left lower abdomen. Clinical examination still remains the first modality used in diagnosing pelvic masses. Imaging modalities like USG, MRI are complimentary in diagnosing pelvic masses \& their origin. Several authors state that the cervicovaginal smear is an inadequate diagnostic tool for the diagnosis of primary fallopian tube carcinoma. Positive Pap smears have been reported in only $0 \%-23 \%$ of cases ${ }^{3}$.Laparotomy \& HPE still remain as the Gold standard for diagnosis. In the above case, USG \& MRI diagnosed an adnexal mass which on laparotomy turned to be a fallopian tube malignancy \& HPE confirmed the diagnosis of tubal malignancy. Tubal malignancy is staged by FIGO into four stages. Prognosis depends upon staging. Asymptomatic cases diagnosed incidentally have 5 year survival rate of $65 \%$. Stage II is $50-60 \%$. Stage III \& IV is $10-20 \%{ }^{10}$.

Therefore women with vague pain abdomen, distention \& abnormal discharge should be subjected to thorough clinical examination, combined with relevant investigations to arrive at proper diagnosis, at the earliest to give better prognosis to the patient.

Staff members, Dept of OBGYN, Dr. PSIMS \& RF

\section{Acknowledgements}

Dept of Radiology, Dr. PSIMS \& RF

Dept. of Pathology, Dr.PSIMS\& RF

\section{References}

[1]. Jonathan S Berek. Ovarian, fallopian Tube and peritoneal cancer.In : Berek\& Novak's Gynaecology $15^{\text {th }}$ edn. WOLTERS KLUWER/LIPPINCOTT WILLIAMS \& WILKINS, 2012.P.1414

[2]. Cohen CJ, Thoas GM, Hagopian GS, Kufe DW, Pollock RE, Holland JF, et al. Neoplasms of the fallopian tube cancer medicine. 5th ed. Canada, B.C: Decker Inc; 2000. p. 1683.

[3]. Takashina T, Ito E, Kudo R. Cytologic diagnosis of primary tubal cancer. ActaCytol1985;29:367-372.

[4]. Rose PG, Shrigley R, Wiesner GL. Germline BRCA2 mutation in a patient with fallopian tube carcinoma: a case report. GynecolOncol 2000;77: 319-320.

[5]. Zweemer RP, van Diest PJ, Verheijen RH et al. Molecular evidence linking primary cancer of the fallopian tube to BRCA1 germline mutations. GynecolOncol 2000;76:45-50.

[6]. Aziz S, Kuperstein G, Rosen B et al. A genetic epidemiological study of carcinoma of the fallopian tube. GynecolOncol 2001;80:341-345.

[7]. Ajithkumar TV, Minimole AL, John MM et al. Primary fallopian tube carcinoma. ObstetGynecolSurv 2005;60:247-252.

[8]. Pfeiffer P, Mogensen H, Amtrup F et al. Primary carcinoma of the fallopian tube. A retrospective study of patients reported to the Danish Cancer Registry in a five-year period. ActaOncol 1989;28:7-11.

[9]. Boutselis JG, Thompson JN. Clinical aspects of primary carcinoma of the Fallopian tube: a clinical study of 14 cases. Am J ObstetGynecol1971;111:98-101.

[10]. Jonathan S Berek. Ovarian, fallopian Tube and peritoneal cancer.In : Berek\& Novak's Gynaecology $15^{\text {th }}$ edn. WOLTERS KLUWER/LIPPINCOTT WILLIAMS \& WILKINS, 2012.P.1416 